\title{
Kinetic Roughening in Two-Phase Fluid Flow through a Random Hele-Shaw Cell
}

\author{
Eduard Pauné and Jaume Casademunt \\ Departament d'Estructura i Constituents de la Matèria, Universitat de Barcelona, Avinguda Diagonal, 647, \\ 08028 Barcelona, Spain
}

(Received 29 July 2002; published 10 April 2003)

\begin{abstract}
A nonlocal interface equation is derived for two-phase fluid flow, with arbitrary wettability and viscosity contrast, $c=\left(\mu_{1}-\mu_{2}\right) /\left(\mu_{1}+\mu_{2}\right)$, in a model porous medium defined as a Hele-Shaw cell with random gap $b_{0}+\delta b$. Fluctuations of both capillary and viscous pressure are explicitly related to the microscopic quenched disorder, yielding conserved, nonconserved, and power-law correlated noise terms. Two length scales are identified that control the possible scaling regimes and which scale with capillary number $\mathrm{Ca}$ as $\ell_{1} \sim b_{0}(c \mathrm{Ca})^{-1 / 2}$ and $\ell_{2} \sim b_{0} \mathrm{Ca}^{-1}$. Exponents for forced fluid invasion are obtained from numerical simulation and compared with recent experiments.
\end{abstract}

DOI: 10.1103/PhysRevLett.90.144504

The displacement of a fluid by another in a porous medium is a problem of fundamental interest in nonequilibrium physics as a paradigm of interface dynamics in disordered media [1,2]. Experiments on bead packs in Hele-Shaw cells [3], in particular, have stimulated considerable theoretical efforts, but the problem has consistently revealed itself to be rather elusive [1,2]. More recently, a new surge of interest has arisen with the recognition of the inherently nonlocal character of the problem as a key ingredient $[4,5]$ and the realization of a new series of experiments in Hele-Shaw cells with random gap [6-8] and with roughened glass plates [9]. Roughening exponents of the proposed nonlocal equations have been explored by means of Flory-type scaling arguments [4] and phase field simulations $[5,6]$. While the specific properties of noise are known to be crucial to determine the universal aspects of interface roughening, fluctuations are usually modeled at a phenomenological level and include only local capillary effects. Noise related to the nonLaplacian viscous pressure due to quenched disorder in the permeability has been so far neglected. While this may be justified for imbibition experiments [5] or, in general, situations close to pinning [10,11], the case of forced fluid invasion does require a quantitative assessment of this point. In addition, it would be desirable to have a unified formulation for general conditions of viscosity contrast $c=\left(\mu_{1}-\mu_{2}\right) /\left(\mu_{1}+\mu_{2}\right)$ and wettability given the rich variety of phenomena that the experimental evidence has unveiled as a function of those parameters [2].

Here we address the general problem of fluid displacement in a Hele-Shaw cell with random gap, as a simple model of a porous medium. This model system has the great advantage that no coarse-graining procedure must be invoked in the theoretical formulation, thus allowing us to derive $a b$ initio a general and complete interface equation, quantitatively accurate, with explicit dependence on "bare" parameters, and including all noise sources. On the experimental side, the system is also appealing since a direct control of the disorder is locally possible on the microscopic scale [6,7].

144504-1 0031-9007/03/90(14)/144504(4)\$20.00
PACS numbers: 47.55.Mh, 05.40.-a, 68.35.Ct

From our microscopic derivation we will get a complete description of fluctuations containing the three basic physical effects of a porous matrix on the interface motion, namely, local variations of (i) capillary pressure, (ii) permeability, and (iii) available volume. Different but not independent noise terms must thus be generated through distinct physical mechanisms from the unique source of randomness. Fluctuating capillary pressure can be directly related to gap variations $\delta b=b-b_{0}$, where $b_{0}$ is the mean value, in terms of the Laplace condition for the pressure jump across the interface

$$
p_{2}-p_{1}=\sigma\left(\kappa+\frac{2 \cos \theta}{b_{0}+\delta b}\right),
$$

where $\kappa$ is the curvature in the cell plane, $\sigma$ is surface tension, and $\theta$ is the contact angle, $\cos \theta=1$ meaning perfect wetting of the invading fluid 1 . The effect of viscous pressure fluctuations, however, is far less obvious due to the inherently nonlocal character of the interplay of (ii) and (iii) in the response of the fluid flow to gap fluctuations. We base our analysis on the assumption that, for sufficiently smooth gap variation (i.e., $|\nabla b| \ll 1$ ), Darcy's law for a Hele-Shaw cell [12] holds locally as

$$
\mathbf{v}=-\frac{\left[b_{0}+\delta b(x, y)\right]^{2}}{12 \mu} \nabla p .
$$

In a capillary tube of lateral size $b$ at fixed injection pressure, from Darcy's law a relative velocity fluctuation scales as $\delta v / v \sim 2 \delta b / b$ (larger permeability implies less resistance to flow) while at fixed flow injection it is exactly the opposite, $\delta v / v \sim-2 \delta b / b$ (mass conservation slows down the flow if there is more volume available). In an actual disordered medium, the solution of the whole pressure field will thus be required to sort out the effective flow conditions at each location. A direct consequence of (iii) is that the $2 \mathrm{D}$ effective flow must give rise in general to a nonconserved interface equation, precisely to account for volume conservation in 3D. In our case, 3D incompressibility implies that the 2D flow will satisfy

(C) 2003 The American Physical Society

144504-1 


$$
\nabla \cdot(b \mathbf{v})=0,
$$

where the gap acts effectively as a variable density.

Equations (2) and (3) imply that the pressure field is non-Laplacian. In order to obtain a closed bulk equation which we can project onto the interface degrees of freedom, we treat the pressure perturbatively in $|\nabla b|$. We thus split the pressure field as $p=p_{0}+\delta p$ and keep only the order $|\nabla b|$ in $\delta p$ to obtain

$$
\begin{aligned}
\nabla^{2} p_{0} & =0, \\
\nabla^{2} \delta p+\frac{3 \nabla b}{b} \nabla p_{0} & =0,
\end{aligned}
$$

where we have neglected higher orders consistently with the fact that they have also been omitted in assuming local Darcy flow. The lowest order Eq. (4a) can be solved as the unperturbed problem $[12,13]$ with the modified boundary condition Eq. (1) which contains all capillary effects, with a simpler boundary condition for the Poisson equation (4b). For $c=1$, this is $\delta p=0$ and yields, in terms of the Laplace Green's function,

$$
\begin{aligned}
& \int_{\text {int }} d s G[x-x(s), y-y(s)] \frac{\partial \delta p}{\partial n} \\
& \quad=-\int d x^{\prime} d y^{\prime} G\left(x-x^{\prime}, y-y^{\prime}\right) \frac{3 \nabla b}{b} \nabla p_{0} .
\end{aligned}
$$

The free-boundary problem is thus defined by Eq. (2) specified at the interface, with $p=p_{0}+\delta p$, and the boundary conditions at infinity. Here we focus on the case of forced fluid invasion, where a fixed velocity $V$ is imposed at infinity and $\mu_{1} \geq \mu_{2}$. We introduce the dimensionless quenched noise $\xi(x, y)$ in the gap spacing $b$ as $b^{2}=b_{0}^{2}[1+\xi(x, y)]$. Noise originated, respectively, from $\delta b$ in Eq. (1), from $\delta b$ in Eq. (2), and from $\delta p$ in Eq. (4b) will be called, respectively, capillary, permeability, and bulk noises.

Concerning the scaling properties of the interface, we are interested in the lowest order approximation on the interface deviation from planarity that is relevant in a renormalization group (RG) sense. Our result for the interface equation for the Fourier transform of the interfacial height $\hat{h}(k)$ for two-fluid displacement under constant injection velocity $V$ takes the form

$$
\begin{aligned}
\frac{1}{V} \frac{\partial \hat{h}(k)}{\partial t}= & \delta(k)-c|k|\left[1+\left(\ell_{1} k\right)^{2}\right] \hat{h}(k)+\mathcal{N}_{h}(k) \\
& -\frac{1}{2}\left(1+\ell_{2}|k|\right) \hat{\xi}_{h}(k)+\hat{\Omega}_{L R}(k, t),
\end{aligned}
$$

where the lengths $\ell_{1}$ and $\ell_{2}$ are defined in terms of the capillary number $\mathrm{Ca}=12\left(\mu_{1}+\mu_{2}\right) V / \sigma$ as $\ell_{1}=$ $b_{0}(c \mathrm{Ca})^{-1 / 2}$ and $\ell_{2}=2 b_{0} \cos \theta \mathrm{Ca}^{-1}$, and where $\mathcal{N}_{h}(k)$ denotes the leading (quadratic) nonlinearities

$$
\begin{gathered}
\mathcal{N}_{h}(k)=-c^{2}|k| \int_{-\infty}^{\infty} d q[1-S(k q)]|q|\left[1+\left(\ell_{1} q\right)^{2}\right] \\
\times \hat{h}(k-q) \hat{h}(q),
\end{gathered}
$$

where $S$ is the sign function. $\hat{\xi}_{h}(k)$ is the Fourier transform of $\xi[x, h(x)]$ and the term $\Omega_{\mathrm{LR}}(k, t)$ is a long-ranged correlated noise of the form

$$
\hat{\Omega}_{\mathrm{LR}}(k, t)=\frac{1}{\mu_{1}+\mu_{2}}\left[\mu_{1} \hat{\Omega}^{-}(k, t)-\mu_{2} \hat{\Omega}^{+}(k, t)\right],
$$

with

$$
\hat{\Omega}^{ \pm}(k, t)=|k| \int d x d y \xi(x, y+V t) e^{-i k x \mp y|k|} \Theta( \pm y),
$$

where $\Theta$ is the step function.

Note that the long-ranged term $\hat{\Omega}_{\mathrm{LR}}(k, t)$ enters effectively as an annealed (explicitly time-dependent) noise (see discussion below). Equations (6)-(9) constitute our central result. Note also that in this formulation we have assumed weak noise so that multiplicative noise terms of order $h \xi$ or nonlinear in $\xi$ have been neglected [14].

The linear deterministic part of Eq. (6) is well known and can be found, for instance, in Refs. $[4,12,13]$. The complete set of deterministic nonlinearities can be obtained systematically using the weakly nonlinear expansion developed in Ref. [13]. Transforming Eq. (7) back to the real space, it is found [13] that quadratic nonlinearities include both nonlocal and local terms. The local terms (in real space) were also considered in Ref. [4] and include the familiar $(\nabla h)^{2}$ term.

The capillary fluctuations give rise to the conserved (area-preserving) noise term proportional to $|k| \hat{\xi}_{h}$. This contribution is associated to the second term in Eq. (1) and is generated adding the noise term to the curvature $\kappa$ and then linearizing, following the scheme used in Ref. [12] or [13]. The bulk noise defined as $\delta v_{\xi} \approx$ $-\left(b_{0}^{2} / 12 \mu\right)(\partial \delta p / \partial n)$ takes the form

$$
\widehat{\delta v}_{\xi}(k)=\frac{3 V}{2}\left[-\hat{\xi}_{h}(k)+\hat{\Omega}_{\mathrm{LR}}(k, t)\right],
$$

as shown below. The $\hat{\xi}_{h}$ term of Eq. (10) adds to the trivial lowest order contribution of permeability noise of the form $V \hat{\boldsymbol{\xi}}_{h}(k)$ and results in the nonconserved noise term present in the interface Eq. (6).

We now proceed to sketch the derivation of the bulk noise. For simplicity, we consider the one-sided case $(c=1)$ [16]. Neglecting orders $\xi \partial_{x} h$, Eq. (5) reads

$$
\begin{array}{r}
\frac{-2}{3} \int_{-\infty}^{\infty} d x^{\prime} \ln \left[\left(x-x^{\prime}\right)^{2}+\left(h(x)-h\left(x^{\prime}\right)\right)^{2}\right] \delta v_{\xi}\left(x^{\prime}\right)=\int_{-\infty}^{\infty} d x^{\prime}\left\{\ln \left[\left(x-x^{\prime}\right)^{2}+\left(h(x)-h\left(x^{\prime}\right)\right)^{2}\right] \xi\left(x^{\prime}, h\left(x^{\prime}\right)\right)\right. \\
\left.+\int_{-\infty}^{h\left(x^{\prime}\right)} d y^{\prime} \frac{2\left(h\left(x^{\prime}\right)-y^{\prime}\right)}{\left(x-x^{\prime}\right)^{2}+\left(h(x)-y^{\prime}\right)^{2}} \xi\left(x^{\prime}, y^{\prime}\right)\right\},
\end{array}
$$

where the integral on $y$ of the right-hand side of Eq. (5) has been integrated by parts and it has been assumed that the noise vanishes at infinity, $\xi(x, y \rightarrow-\infty)=0$. It has also been applied that $(\nabla b / b) \nabla p_{0} \simeq-\left(6 \mu / b_{0}^{2}\right)(\partial \xi / \partial y) V$ and that 
the Green function for the Laplace equation for infinite space has the form $G(x, y)=-(4 \pi)^{-1} \ln \left[x^{2}+y^{2}\right]$. With the change $y=y^{\prime}-V t$, and to lowest order in the interface deviation $h-V t$ with respect to the mean interface position, that is, considering $h-V t \simeq 0$ except in the rapidly varying function $\xi(x, h)$, Eq. (11) then reads

$$
\int_{-\infty}^{\infty} d x^{\prime} \ln \left|x-x^{\prime}\right| \delta v_{\xi}\left(x^{\prime}\right)=-\frac{3}{2} \int_{-\infty}^{\infty} d x^{\prime}\left\{\ln \left|x-x^{\prime}\right| \xi\left(x^{\prime}, h\right)+\int_{-\infty}^{0} d y \frac{-y}{\left(x-x^{\prime}\right)^{2}+y^{2}} \xi\left(x^{\prime}, y+V t\right)\right\} .
$$

To solve explicitly the above equation we Fourier transform it and use [17] $\mathcal{F}[\ln |x|](k)=-\pi^{-1}|k|^{-1}$ $2 \pi(\gamma+\ln 2 \pi) \delta(k)$, where $\gamma$ is Euler's constant. Using this expression, Eq. (11) is obtained (for $c=1$, i.e., $\mu_{2}=0$ ).

The quenched noise will be typically correlated on a microscopic scale $a$. For $k a \ll 1, \xi$ is effectively white. If $\left\langle\xi(x, y) \xi\left(x^{\prime}, y^{\prime}\right)\right\rangle=\Delta \delta\left(x-x^{\prime}\right) \delta\left(y-y^{\prime}\right)$, then we have

$$
\left\langle\hat{\Omega}_{\mathrm{LR}}(k, t) \hat{\Omega}_{\mathrm{LR}}\left(k^{\prime}, t^{\prime}\right)\right\rangle=\frac{\Delta}{2 \pi}|k| \delta\left(k+k^{\prime}\right) e^{-|k| V\left|t-t^{\prime}\right|},
$$

so $\hat{\Omega}_{\mathrm{LR}}$ scales as $|k|^{1 / 2}$ and introduces long-range memory. Accordingly, low- $k$ behavior is dominated by the local part of bulk noise. This implies that 3D conservation overcomes permeability at low $k$, giving rise to an overall nonconserved noise with the same sign as the capillary noise. Although direct computation of both terms in the bulk noise shows that the local part is typically larger, it is unclear to what extent neglecting the long-range term may miss important details of local interface pinning which may eventually affect the scaling. Furthermore, for $k a \sim 1$, both local and nonlocal parts of the bulk noise are comparable but then the annealing approximation may not be justified, and one must rely on Eq. (11). The local and nonlocal contributions to bulk noise may be of the same order in other situations. For instance, persistent noise $\xi=\xi(x)$ yields $\delta v_{\xi}=0$, with nonconserved and conserved noises opposing each other.

One of the salient features of Eq. (6) is that the problem has two characteristic length scales. $\ell_{1}$ controls the wellknown crossover between (deterministic) capillary and viscous forces [5]. The second length $\ell_{2}$ is a newly identified one which defines a crossover between conserved and nonconserved noise. For general viscosities and wetting conditions, the two length scales are arbitrary and define a variety of possible scaling regimes and crossovers depending on their relative size. Experiments of a wetting fluid invading an inviscid one $(\cos \theta=1, c=1)$ typically have $\ell_{2} \gg \ell_{1}$. Concerning the nonlinearities, power counting arguments predict that quadratic terms are relevant in the RG sense only if the viscous term $c|k|$ is absent, that is, for $|k| \ell_{1} \gg 1$. This capillary-dominated regime will always be observed at large $k$ and short times provided $\ell_{2} \gg \ell_{1}$. A crossover to the viscous-dominated regime will eventually occur for $|k| \ell_{1} \sim 1$ and then the quadratic nonlinearities do become irrelevant. A second crossover within the viscous regime, from conserved to nonconserved noise, will occur for $|k| \ell_{2} \sim 1$.

Experiments of random-gap cells [8], and presumably other forced fluid invasion experiments [3], operate at $\ell_{2} \gg \ell_{1}$. From Eq. (6), the relevant equation in this capillary regime reduces to

$$
\frac{1}{V} \frac{\partial \hat{h}(k)}{\partial t}=\delta(k)-|k|\left[\left(\tilde{\ell}_{1} k\right)^{2} \hat{h}(k)+\frac{1}{2} \ell_{2} \hat{\xi}_{h}(k)\right]
$$

with $\tilde{\ell}_{1}=b_{0} \mathrm{Ca}^{-1 / 2}$. Nonlinear terms and the nonlocal part of bulk noise have also been neglected on the basis of direct numerical computation. In fact, the knowledge of the bare coefficients of nonlinear and noise terms has allowed us to assess the quantitative importance of both contributions. While the nonlocal noise term remains negligible (except for large $k$ ), the effect of nonlinearities, although RG-relevant, remained also negligible for times within the duration of the capillary regime. Since the quadratic nonlinearities in Eq. (6) vanish exactly for $c=0$, we may refer to the growth described by Eq. (14) as the universality class of "symmetric fluid invasion" (SFI).

We now study the SFI scaling by numerical simulation of Eq. (14). The quantities of interest concerning the scaling properties are the root mean square of the interface height fluctuations, $W$, and the structure factor $S(k, t)$. The width grows with time as $W(t) \sim t^{\beta}$ before saturation, and the saturation width scales with system size $L$ as $W_{\text {sat }} \sim L^{\alpha}$. The roughness exponent $\alpha$ can also be obtained from the relation $S(k, t) \sim k^{-1-2 \alpha}$ for long times. For further reference, we provide the scaling exponents of Eq. (14) for two cases that are exactly solvable [15], namely, for persistent noise $\xi=\xi(x)$ and annealed noise $\xi=\xi(x, t)$ which, assuming $\delta$ correlations, give respectively $\alpha=3 / 2, \beta=1 / 2$ and $\alpha=0, \beta=0$.

Typical results for Eq. (14) are shown in Fig. 1. We choose $V=1, a=0.0625, \ell_{1}=50$, and $\ell_{2}=3000$, which satisfy the criterion $a \ll \ell_{1} \ll \ell_{2}$. For these values, we have obtained a roughness exponent $\alpha=1.2 \pm$ 0.05 (very close to the value $\alpha=1.25$ reported in Ref. [5]) and a growth exponent $\beta=0.68 \pm 0.02$. The scaling of the height-height correlation function has been found to be fully consistent with $\alpha_{\text {loc }}=1$, as expected from the superrough value of $\alpha>1$. Note that the scaling of the power spectrum $S(k, t)$ at large $k$ corresponds to the case of persistent noise, $\alpha=3 / 2$ : short segments of the interface "feel" effectively a persistent noise for short times.

Increasing by an order of magnitude the value of $V$ with the corresponding variation of $\ell_{1}$ and $\ell_{2}$ does modify the scaling behavior: for low values of $k$ the scaling of $S(k, t)$ yields $\alpha \simeq 0$, the value obtained for annealed noise, while 


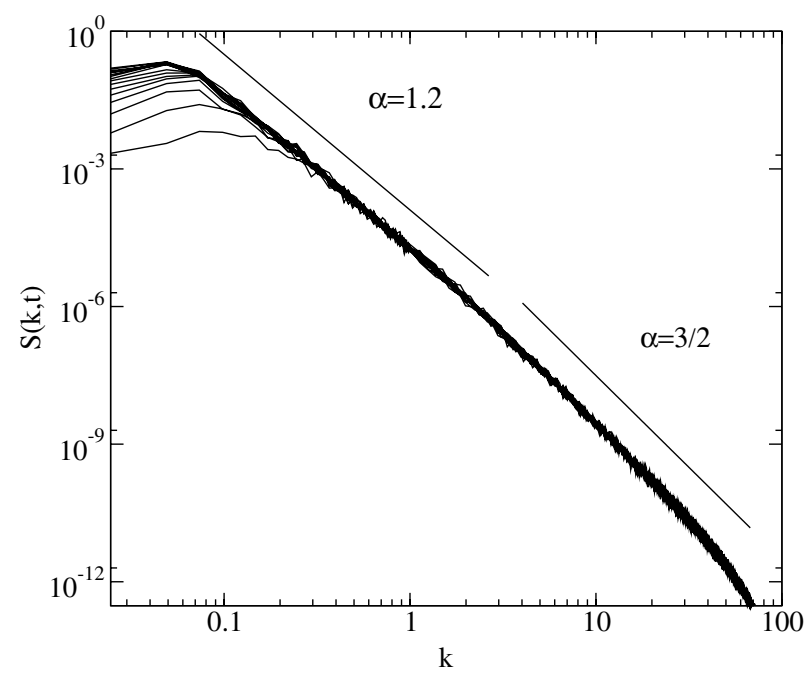

FIG. 1. Structure factor for a system with $L=256$. The data are for $t=0.5$ (lower curve) to $t=12.0$, and time interval $\Delta t=0.5$. The straight line with slope $-3.3(\alpha=1.2)$ is a fit, and the other straight line has slope $-4(\alpha=3 / 2)$.

for larger $k$ the observed scaling is essentially the same as in Fig. 1. Hence, the noise acting on the interface at low $k$ for large $V$ is effectively annealed. The value of the exponent $\beta$ is observed to decrease with increasing $V$, consistent with the trend towards an effective annealed noise, for which $\beta=0$. On the other hand, if $V$ is decreased by an order of magnitude, the scaling observed in Fig. 1 is essentially unchanged.

The values of $\mathrm{Ca}$ and $V$ we have used are of similar magnitude to the ones of the experimental work of Soriano et al. [8]. They studied forced fluid invasion in Hele-Shaw cells with variable gap, and therefore our interface Eq. (6) should describe their results except, maybe, for effects related to the sharpness of edges (gap discontinuities), excluded in our derivation. According to our analysis, their experimental parameters belong to the capillary regime Eq. (14) of Eq. (6). The exponents reported in Ref. [8] for large injection rates are $\alpha \simeq 0$ for small $k$ and $\alpha \simeq 1.3$ for large $k$ are fully consistent with our results for large $V$. However, this is not the case for small values of $V$. This discrepancy can clearly be attributed to the sharp-edge anchoring effects mentioned above.

In summary, we have derived a general interface equation for a random Hele-Shaw cell, including all nonlocal effects and long-range correlations in the noise terms. The explicit knowledge of all contributions originated from the gap randomness, including all coefficients in terms of known physical parameters, has revealed crucial for a quantitative assessment of the different effects in distinct regimes. In particular, we have identified a universality class which is relevant to existing experiments and obtained explicit values for exponents. We expect that the physical insights and the predictive power of the theoretical framework here presented may be useful to reinterpret data and design new experiments, in particular, in the yet unexplored ranges of parameters such as viscosity contrast.

We are grateful to Otger Campàs for helpful discussions. Financial support from DGES (Spain) under Project No. BXX2000-0638-C02-02 is acknowledged.

[1] A.-L. Barabási and H. Stanley, Fractal Concepts in Surface Growth (Cambridge University Press, Cambridge, UK, 1995).

[2] T. Halpin-Healy and Y.-C. Zhang, Phys. Rep. 254, 215 (1995); J. Krug, Adv. Phys. 46, 139 (1997).

[3] M. A. Rubio, C. A. Edwards, A. Dougherty, and J. P. Gollub, Phys. Rev. Lett. 63, 1685 (1989); V. K. Horváth, F. Family, and T. Vicsek, J. Phys. A 24, L25 (1991); S. He, G. L. M. K. S. Kahanda, and P.-z. Wong, Phys. Rev. Lett. 69, 3731 (1992).

[4] V. Ganesan and H. Brenner, Phys. Rev. Lett. 81, 578 (1998).

[5] M. Dubé et al., Phys. Rev. Lett. 83, 1628 (1999); Eur. Phys. J. B 15, 701 (2000).

[6] A. Hernández-Machado et al., Europhys. Lett. 55, 194 (2001).

[7] J. Soriano et al., Phys. Rev. Lett. 89, 026102 (2002).

[8] J. Soriano, J. Ortín, and A. Hernández-Machado, Phys. Rev. E 66, 031603 (2002).

[9] D. Geromichalos, F. Mugele, and S. Herminghaus, Phys. Rev. Lett. 89, 104503 (2002).

[10] L.-H. Tang and H. Leschhorn, Phys. Rev. A 45, R8309 (1992).

[11] L.-H. Tang, M. Kardar, and D. Dhar, Phys. Rev. Lett. 74, 920 (1995).

[12] J. Casademunt, D. Jasnow, and A. Hernández-Machado, Int. J. Mod. Phys. B 6, 1647 (1992).

[13] E. Alvarez-Lacalle, J. Casademunt, and J. Ortín, Phys. Rev. E 64, 016302 (2001).

[14] Terms of this type coming from capillary and permeability can be included systematically [15], but those from the bulk cannot be obtained explicitly.

[15] E. Pauné and J. Casademunt (unpublished); E. Pauné, $\mathrm{Ph}$.D. thesis, University of Barcelona.

[16] The derivation for $c \neq 1$ uses the formulation of Ref. [12] but is more involved and will be presented elsewhere [15].

[17] I. Richards and H. Youn, Theory of Distributions: A NonTechnical Introduction (Cambridge University Press, Cambridge, UK, 1990). 